\title{
Draft Genome Resources Sequences of Six Pseudomonas syringae pv. actinidiae Strains Isolated from Actinidia chinensis var. deliciosa Leaves in Portugal
}

\author{
Aitana Ares, ${ }^{1,2}$ Marta Tacão, ${ }^{3}$ Daniela Figueira, ${ }^{1,2}$ Eva Garcia, ${ }^{1,2}$ and Joana Costa ${ }^{1,2, \dagger}$ \\ ${ }^{1}$ University of Coimbra, Centre for Functional Ecology, Department of Life Sciences, Calçada Martim de \\ Freitas, 3000-456 Coimbra, Portugal \\ ${ }^{2}$ FitoLab, Laboratory for Phytopathology, Instituto Pedro Nunes, 3030-199 Coimbra, Portugal \\ ${ }^{3}$ CESAM, Department of Biology, University of Aveiro, Portugal
}

\begin{abstract}
Pseudomonas syringae pv. actinidiae is a quarantine bacterium affecting all the Portuguese main areas of kiwifruit production. We report the draft genome of six $P$. syringae pv. actinidiae strains isolated from symptomatic leaves of Actinidia chinensis var. deliciosa in a study that determined the genetic population structure of the endophytic and epiphytic populations in two consecutive seasons. Average nucleotide identity values were above $99 \%$ similarity with reference strains from $P$. syringae pv. actinidiae biovar 3 . The genomic differences found between these strains confirm the genetic diversity described for $P$. syringae pv. actinidiae population in Portugal. Furthermore, data provide evidence that the initial clonal expansion of $P$. syringae pv. actinidiae in Europe was followed by a genomic diversification constituting a valuable resource for epidemiological and evolutionary studies, namely when adopting strategies for epidemics management.
\end{abstract}

\section{Genome Announcement}

In 2010, Pseudomonas syringae pv. actinidiae, the causal agent of bacterial canker of kiwifruit, was detected on 2-year-old plants of Actinidia chinensis var. deliciosa 'Summer' in kiwifruit orchards in the northwest of Portugal (Balestra et al. 2010). The Portuguese P. syringae pv. actinidiae isolates belong to the pandemic biovar 3, responsible for substantial economic losses worldwide (Garcia et al. 2018; Moura et al. 2015; Renzi et al. 2012). Initially, this pathogen was part of European and Mediterranean Plant Protection Organization (EPPO) Alert A1 List, but from 2012 the EPPO included $P$. syringae pv. actinidiae in the EPPO A2 List and is considered a major threat and the main cause of economic losses for Actinidia cultivation worldwide (EPPO 2012). Nevertheless, the characterization of $P$. syringae pv. actinidiae populations found in Portugal is limited and sometimes contradictory. While Renzi et al. (2012) revealed a genetic uniformity in strains isolated in Portugal during 2011, further studies revealed some genetic variability (Cruz et al. 2014; Garcia et al. 2018; Moura et al. 2015).

In this study, we described six draft genomes of $P$. syringae pv. actinidiae isolates representative of distinct genetic populations based on BOX profiles (Figueira et al. 2020). These populations were identified in a previous study that characterized the genetic structure of the endophytic and epiphytic population of $P$. syringae pv. actinidiae from a culture collection of 600 isolates recovered from leaves of Actinidia chinensis var. deliciosa from four kiwifruit orchards characterized by distinct abiotic conditions in two consecutive seasons (Figueira et al. 2020). Strain KW1361 represents a $P$. syringae pv. actinidiae population found in all orchards, in spring

${ }^{\dagger}$ Corresponding author: J. Costa; jcosta@uc.pt

The author(s) declare no conflict of interest.

Accepted for publication 2 June 2020.
Funding

This work was supported by Action 1.1 Operational Groups promoted by PDR2020 and co-financed by EAFRD, within the framework of Portugal 2020 through the project i9K (PDR2020-101031204). Thanks are due to Fundação para a Ciência e a Tecnologia/MCTES for the financial support of CFE (UID/BIA/ 04004/2020) and CESAM (UIDP/50017/ $2020+$ UIDB/50017/2020) through national funds. Fundação para a Ciência e a Tecnologia supported the contract research of J. Costa (SFRH/BPD/ 112157/2015) and M. Tacão (CEEC/ 01304/2017). A. Ares acknowledges financial support from FCT/MEC through national funds and the co-funding by the FEDER, within the PT2020 Partnership Agreement and COMPETE2020 within the project UID/BIA/04004/2013.

\section{Keywords}

Actinidia chinensis, bacteriology, disease control and pest management, draft genome, ecology and epidemiology, genetics and resistance, kiwi diseases, Pseudomonas syringae pv. actinidiae 
Table 1. General features of the whole genome sequences of Pseudomonas syringae pv. actinidiae strains

\begin{tabular}{lccrrrr} 
Features & KW83 & KW98 & KW503 & KW1361 & KW1483 & KW1606 \\
Accumulated length (Mb) & 6.22 & 6.20 & 6.16 & 6.22 & 6.22 & 5.22 \\
GC content (\%) & 58.5 & 58.5 & 58.5 & 58.5 & 58.5 \\
Number of contigs & 303 & 301 & 298 & 297 & 300 \\
N50 & 41,296 & 41,322 & 42,568 & 42,568 & 41,955 \\
Predicted CDS & 5,982 & 5,945 & 5,872 & 5,964 & 5,968 & $120 \times$ \\
Coverage & $143 \times$ & $143 \times$ & $160 \times$ & 5,899 & $178 \times$ & $130 \times$ \\
Sequenced reads & $5,929,000$ & $5,918,580$ & $6,551,020$ & $7,372,490$ & $4,974,440$ \\
Sequenced bases & $1,778,700,000$ & $1,775,574,000$ & $1,965,306,000$ & $2,211,747,000$ & $1,492,332,000$ & $1,622,047,800$ \\
Number of RNAs & 56 & 56 & 57 & 55 & 54 \\
\hline
\end{tabular}

and autumn, and as epiphytic and endophytic. Strains KW83, KW98, and KW503 represent populations exclusively detected in spring, both as epiphytic and endophytic. On the other hand, strain KW1483 represents a population recovered from two orchards exclusively as an endophyte, both in spring and autumn. Finally, strain KW1606 belongs to an endophytic population found in a single plant and only in autumn. The population represented by strains KW98, KW1361, and KW1483 coexisted during spring as endophytes in a single plant.

The analyzed $P$. syringae pv. actinidiae strains were recovered from Actinidia chinensis var. deliciosa 'Summer' symptomatic leaves by isolation in King's B medium at $25^{\circ} \mathrm{C}$ for $48 \mathrm{~h}$. Identification was performed according to Gallelli et al. (2011). From each strain, the genomic DNA was extracted using NZY Microbial gDNA Isolation kit (Nzytech), following the manufacturer's instruction and outsourced to Eurofins Genomics (Konstanz-Germany) to perform the sequencing using Sequencer Illumina HiSeq platform with the NovaSeq 6000 S2 PE150 XP system and prepare the standard genomic library. The raw sequence data generated were processed for quality with FastQC (Andrews 2010), filtered with an average of 6,025,392 reads, de novo assembled as contigs using SPAdes 3.12.0 (Bankevich et al. 2012), and annotated with the automated service RAST (rapid annotation using subsystem technology, http://rast.nmpdr.org).

General features of the six genomes are detailed in Table 1. The characteristics were similar to other $P$. syringae pv. actinidiae strains deposited in the National Center for Biotechnology Information (NCBI) database. All strains presented a 58.5\% GC content, and the accumulated length varied from 6.16 to $6.22 \mathrm{Mb}$. They comprised between 297 to 303 contigs, and the number of predicted coding sequences for each genome varied from 5,945 to 5,982 (Table 1). The number of RNAs predicted by RAST also was different between strains with 54 RNAs for KW1483 and KW1606, 55 RNAs for KW1361, 56 RNAs for KW83 and KW98, and 57 RNAs for KW503.

These data provide evidence to support the genomic diversification events in $P$. syringae pv. actinidiae populations following the initial clonal expansion in Europe. This is vital to understand kiwifruit bacterial canker disease occurrence and $P$. syringae pv. actinidiae evolution, namely when adopting strategies for epidemic management.

Accession numbers. The genome sequences have been deposited as GenBank accession numbers SAMN14770793 (KW83), SAMN14770794 (KW98), SAMN14770795 (KW503), SAMN14770796 (KW1361), SAMN14770797 (KW1483), and SAMN14770798 (KW1606).

\section{Acknowledgments}

We thank the Associação Portuguesa de Kiwicultores and the producers that collaborated in this work.

\section{Literature Cited}

Andrews, S. 2010. Fast QC: A quality control tool for high throughput sequence data. Babraham Bioinformatics. http://www.bioinformatics.babraham.ac.uk/projects/fastqc/

Balestra, G. M., Renzi, M., and Mazzaglia, A. 2010. First report of bacterial canker of Actinidia deliciosa caused by Pseudomonas syringae pv. actinidiae in Portugal. New Dis. Rep. 22:10.

Bankevich, A., Nurk, S., Antipov, D., Gurevich, A., Dvorkin, M., Kulikov, A. S., Lesin, V., Nikolenko, S., Pham, S., Prjibelski, A., Pyshkin, A., Sirotkin, A., Vyahhi, N., Tesler, G., Alekseyev, M. A., and Pevzner, P. A. 2012. SPAdes: A new genome assembly algorithm and its applications to single-cell sequencing. J. Comput. Biol. 19:455-477.
Cruz, L., Cruz, J., Fernandes, C., Chicau, G., and Tenreiro, R. 2014. Bacterial canker of kiwi caused by Pseudomonas syringae pv. actinidiae in Portugal-Disease importance and pathogen characterization. SCAP-SPF Symposium.

EPPO. 2012. European and Mediterranean Plant Protection Organization A1 and $A 2$ lists of pests recommended for regulation as quarantine pests. https://www.eppo.int/ACTIVITIES/plant_quarantine/A2_list

Figueira, D., Garcia, E., Ares, A., Igor, T., Veríssimo, A., and Costa, J. 2020. Genetic diversity of Pseudomonas syringae pv. actinidiae: Seasonal and spatial population dynamics. Microorganisms 8:931. 
Gallelli, A., Talocci, S., L'Aurora, A., and Loreti, S. 2011. Detection of Pseudomonas syringae pv. actinidiae, causal agent of bacterial canker fruit, from symptomless fruits and twigs, and from pollen. Phytopathol. Mediterr. 50:462-472.

Garcia, E., Moura, L., Abelleira, A., Aguín, O., Ares, A., and Mansilla, P. 2018. Characterization of Pseudomonas syringae pv. actinidiae biovar 3 on kiwifruit in north-west Portugal. J. Appl. Microbiol. 125:1147-1161.
Moura, L., Garcia, E., Aguín, O., Ares, A., Abelleira, A., and Mansilla, P. 2015. Identification and characterization of Pseudomonas syringae pv. actinidiae (Psa) in Entre Douro e Minho region (Portugal). Rev. Cienc. Agrar. (Lisb.) 38:196-205.

Renzi, M., Mazzaglia, A., and Balestra, G. M. 2012. Widespread distribution of kiwifruit bacterial canker caused by the European Pseudomonas syringae pv. actinidiae genotype in the main production areas of Portugal. Phytopathol. Mediterr. 51:402-409. 